\title{
Les répertoires subtils d'un terrain contaminé
}

\section{Sophie Houdart}

\section{OpenEdition}

Journals

Édition électronique

URL : https://journals.openedition.org/tc/8567

DOI : $10.4000 /$ tc. 8567

ISSN : 1952-420X

\section{Éditeur}

Éditions de l'EHESS

\section{Édition imprimée}

Date de publication : 18 décembre 2017

Pagination : 88-103

ISBN : 978-2-7132-2708-0

ISSN : 0248-6016

\section{Référence électronique}

Sophie Houdart, «Les répertoires subtils d'un terrain contaminé », Techniques \& Culture [En ligne], $68 \mid$ 2017, mis en ligne le 18 décembre 2019, consulté le 29 septembre 2022. URL : http:// journals.openedition.org/tc/8567 ; DOI : https://doi.org/10.4000/tc.8567 



\title{
Les répertoires subtils d'un terrain contaminé
}

\author{
«Ça sent le silence. Il est lourd.» \\ Furukawa 2013: 69 .
}

Printemps 2015. Département de Fukushima, Japon. Minami-Sôma, village d'Ôdaka à deux kilomètres du barrage routier qui marque l'entrée de la zone interdite ${ }^{1}$. Arrivée chez Monsieur Nemoto, agriculteur, en début d'après-midi. Plusieurs personnes sont déjà sur place, entourant Monsieur Nemoto comme on le ferait pour un témoin précieux. J'en reconnais certains, déjà présents deux ans plus tôt pour le repiquage du riz. Une équipe de journalistes accompagne le groupe. Au total nous sommes une petite vingtaine. Dans la cour, sur l'herbe, un faisan magnifique (kiji) picore nonchalamment. La scène est inhabituelle; l'oiseau n'a manifestement pas l'intention de bouger, et tout le monde l'observe s'affairer comme si nous n'étions pas là. Deux rangées de baquets ont été alignées sur le sol. Sur les baquets, une fine couche de terre. Dans la première rangée, nous éparpillerons des graines de mochigomi, dans la seconde des graines de ten no butsu, une variété locale très prisée - ten no butsu, cela veut dire « graines du ciel ». Éparpiller les graines. Recouvrir d'une nouvelle couche de terre. Tout le monde s'y met -nous sommes bien plus nombreux qu'il ne faut pour remplir la tâche. Nous opérons en silence et de manière très consciencieuse. On s'applique, on lisse, on bichonne, on perfectionne. On a le temps. Monsieur Nemoto nous moque d'ailleurs gentiment, nous montrant le geste - de grandes lancées, un geste ample - alors que nous faisons les choses avec minutie et retenue. Pour finir, c'est Monsieur Nemoto qui arrose les baquets. La scène est filmée, enregistrée par les journalistes, long micro au bout de la perche, caméra à l'épaule. Photographiée aussi par toutes les personnes présentes. Membres d'association d'agriculture biologique ou de défense de l'agriculture tout court, journalistes, amis de la famille: nous sommes tous là - moi exactement au même titre que les autres - pour attester, en cette période du repiquage du riz, que quelque 
chose se passe bel et bien: quelque chose de l'ordre de l'échange (nous nous photographions les uns les autres), de l'ordre de la transmission, de l'ordre de la vie ou de l'espoir de la vie à renaître des graines du ciel - quoique d'une vie d'une teneur différente. Le faisan tient parfaitement son rôle dans la scène. Il est d'ailleurs lui aussi photographié, et même filmé par les journalistes qui passent un long moment à trouver le bon angle pour le saisir - le bon angle, c'est celui qui fait apparaître l'habitation de Monsieur Nemoto derrière le faisan. Le faisan avec pour fond la végétation et la colline n'aurait évidemment pas produit le même effet: il n'aurait pas rendu l'incongruité de la situation, un grand oiseau si proche des habitations délaissées, ayant manifestement fait sien ce bout de territoire.

Comme deux années auparavant, nous partons ensuite ensemble jusqu'aux terres de Monsieur Nemoto, son champ, la zone potagère. Au bord de la rizière, un autre faisan fait entendre son cri. Un pic-vert au loin. L'endroit est d'un calme particulier, d'un silence très dense. Nous passons les plans de culture en revue, là les poireaux, là les salades que nous goûtons, les fleurs. Là encore les carottes, que Monsieur Nemoto nous invite à déterrer. Chacun repartira avec quelque chose, certains rempliront des sacs. On peut y aller généreusement, ce qui sera laissé sur place profitera une nouvelle fois aux sangliers, malgré le fil électrique qui protège bon an mal an le potager. Certaines rangées, pour l'instant vides, ont été laissées à des équipes universitaires, d'autres à des associations, à des fins expérimentales. Dans un des coins, l'université de Fukushima a installé un filtre pour contrôler les poussières qui viennent encore de la centrale. Je retrouve la même atmosphère qu'il y a deux ans, joviale; les tons enjoués et les notes admiratives de ce que la terre est susceptible de donner - même dans ces conditions.

En retrait de la Route 6, qui longe la côte du Pacifique, la maison de Monsieur Nemoto n'est qu'à quelques kilomètres d'étendues dévastées, mais ici, les maisons sont restées debout, la chaussée n'a pas été endommagée. Reste ce silence. Suspicion. Si j'omettais les raisons pour lesquelles je suis venue ici, je serais probablement renvoyée à la question: que s'est-il donc passé ici? Que s'est-il donc passé qui laisse ce territoire, ce paysage, à ce point aphones? Je vois bien les habitations plus ou moins parsemées, les routes et les carrefours, j'aperçois même la voie ferrée en surplomb - mais je n'entends aucun des bruits qui leur sont à chacun associés: bruits de fréquentation ou de circulation, bruits de moteur ou de frein, bruit de train. Mais présence persévérante de ce faisan silencieux, écho disproportionné du pic-vert frappant l'arbre. Oui, bel et bien, «ça sent le silence» et il est «lourd».

Plusieurs témoignages restituent ainsi la texture sonore propre à ces espaces désertés, post-catastrophiques. On n'entendait aucun oiseau, diront certains. On n'entendait justement qu'un seul oiseau, diront d'autres. Un trop peu ou un trop-plein qui sonnent l'un et l'autre inhabituels. Dans les deux cas, ils marquent une saillance inquiétante qui fait sentir combien quelque chose s'est passé là, difficile à qualifier, mais qui continue de résonner dans l'air. Au cas où on l'aurait oublié, quatre années plus tôt, en mars 2011, un séisme d'une envergure telle qu'il déclenchait un tsunami qui générait lui-même une catastrophe nucléaire avait eu lieu non loin de chez Monsieur Nemoto. Depuis, Monsieur Nemoto se rendait presque quotidiennement chez lui, dans une zone dite « de préparation au retour», ouvrait sa maison au matin, entretenait 
son potager, défrichait les bords de chemin, semait, puis à la nuit tombée, il refermait la maison et rejoignait l'un des groupes de logements provisoires à des dizaines de kilomètres de là. Il s'accrochait fort pour ne pas devenir un simple visiteur de sa propre habitation, de ses propres terres, et continuer de donner du sens à une réalité vacillante. Être amené à écrire «au cas où on l'aurait oublié » ne relève pas d'une manœuvre stylistique destinée à accentuer ou rappeler le contexte dans lequel sont menées ces observations - c'est prendre acte, par cette simple opération langagière, de la double situation dans laquelle tout un chacun se trouve. D'abord, nos sens humains ne nous permettent pas d'appréhender la catastrophe nucléaire; toute conversation ou toute observation sur les opérations de mesure de la radioactivité le rappellent, qui s'ouvrent invariablement sur l'idée que celle-ci ne se voit pas, ne s'entend pas, ne se touche pas. Rien ne la rend manifeste aux sens humains. Pour le dire autrement, nous ne sommes pas équipés pour la percevoir. Ensuite, les politiques et les institutions qu'elles soutiennent travaillent avec hardiesse à nous en faire oublier les conséquences. Très vite après la catastrophe, les côtes ont été déblayées, les fissures comblées, les routes sont à nouveau devenues accessibles. Bientôt, la plupart des villages dont les gens ont été déplacés seront à nouveau habités, ne serait-ce qu'en partie; d'autres auront été construits ${ }^{2}$. Autrement dit, il n'y aura bientôt plus rien à voir et l'inquiétant silence aura lui-même été recouvert par les bruits familiers.

Faut-il se résoudre à «oublier Fukushima» (Filine 2012)? Je soutiens que s'il est quelque chose que l'on puisse faire, au contraire, c'est se préparer, suivant l'invitation du philosophe Didier Debaise, à «intensifier le sens de l'importance », de manière à continuer à «éprouver et [à] sentir ce qui importe dans [cet événement] (Debaise 2015: 132-133). Comment procéder? Dans ce qui suit, je m'essayerai à plusieurs choses. Comme en atteste l'entrée en matière qui ouvre cet article, je tenterai pour une part de coller au plus proche de situations vives, dans lesquelles se lit, parfois de manière échevelée, l'imbroglio sensible auquel est soumis tout observateur attentif d'existences malmenées par une catastrophe nucléaire. Je m'adosserai pour ce faire à plusieurs histoires au moyen desquelles je chercherai, par leur contiguité, à rendre les liaisons internes entre des événements qui auraient pu être tenus pour disparates. Suivant l'invitation de Donna Haraway de «rester avec le trouble» (Haraway 2016) par laquelle elle incite à mettre en récit les possibilités de «cohabitation, de coévolution, de socialité interspécifique » entre des êtres hétérogènes (2010), je choisirai, dans ces histoires, de maintenir le trouble plutôt que de le résorber, de m'installer dans la situation d'inconfort qu'il signale, de le prolonger même afin de comprendre la nature de ce qui y est mis en rapport - cela, «sans demander à une théorie ou à un principe de définir une position "innocente", n’ayant pas à "répondre" pour ses conséquences» (Stengers 2014). À vrai dire, le terrain lui-même ne me laisse pas trop le choix, en me confrontant aux limites de mon ethnographie et de mon écriture: à la manière d'Anna Tsing, dont le livre The Mushroom at the End of the World est conçu comme « un assemblage ouvert et non une machine logique» (Tsing 2015: viii), la mise en récit s'est imposée ici comme un discours adéquat à la réalité complexe de Fukushima et à la « déprise cognitive» (Moreau 2017: 69) qui m’accablait. Je cultiverai d'autre part une forme de tiraillement volontaire en allant chercher du côté de l'histoire du compteur Geiger de quoi alimenter ma réflexion sur le type de calibrage sensible (Dettelbach 
L'apprêt des graines de riz avant le repiquage.

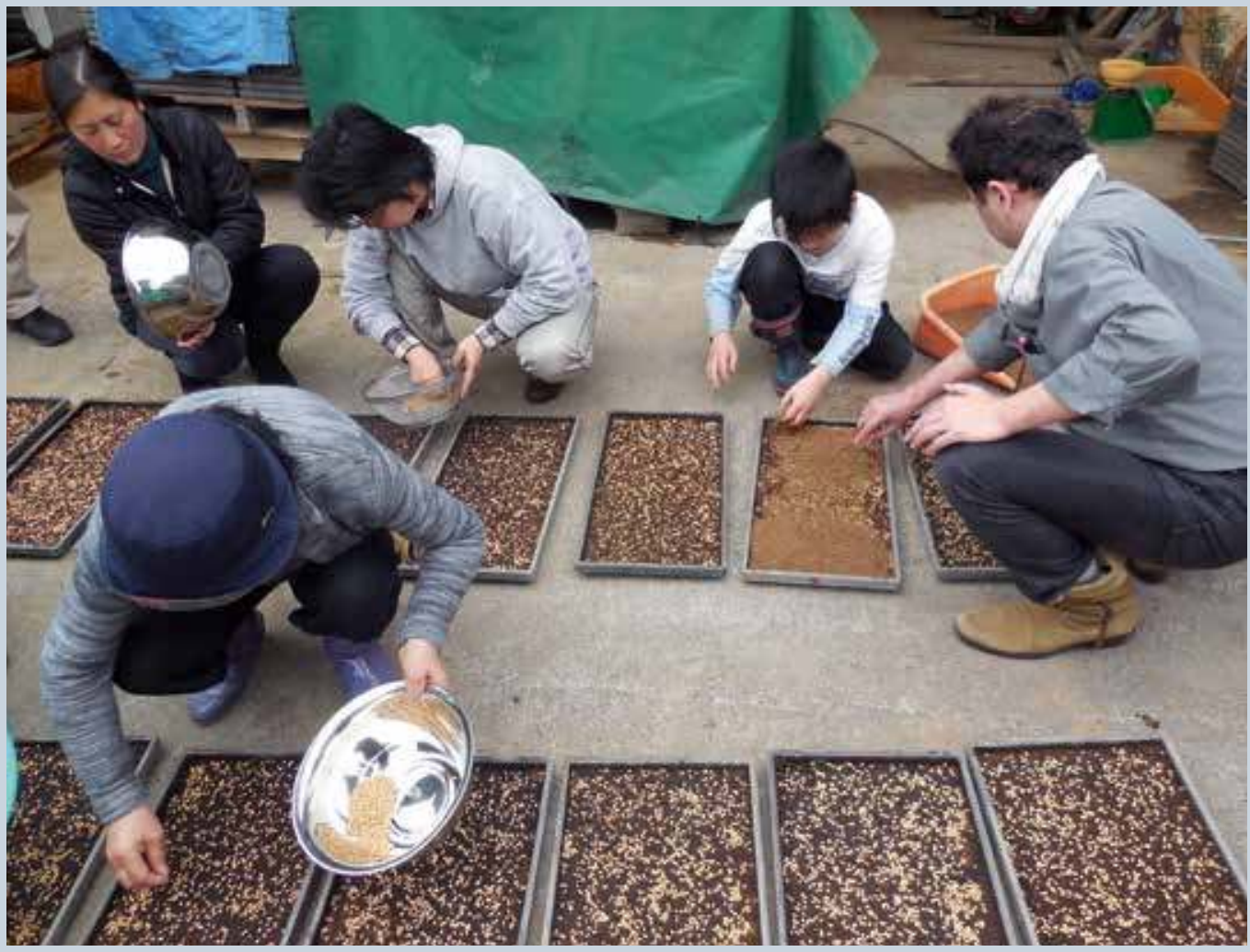

2. Chez Monsieur Nemoto, les médias attrapent la présence incongrue du faisan.

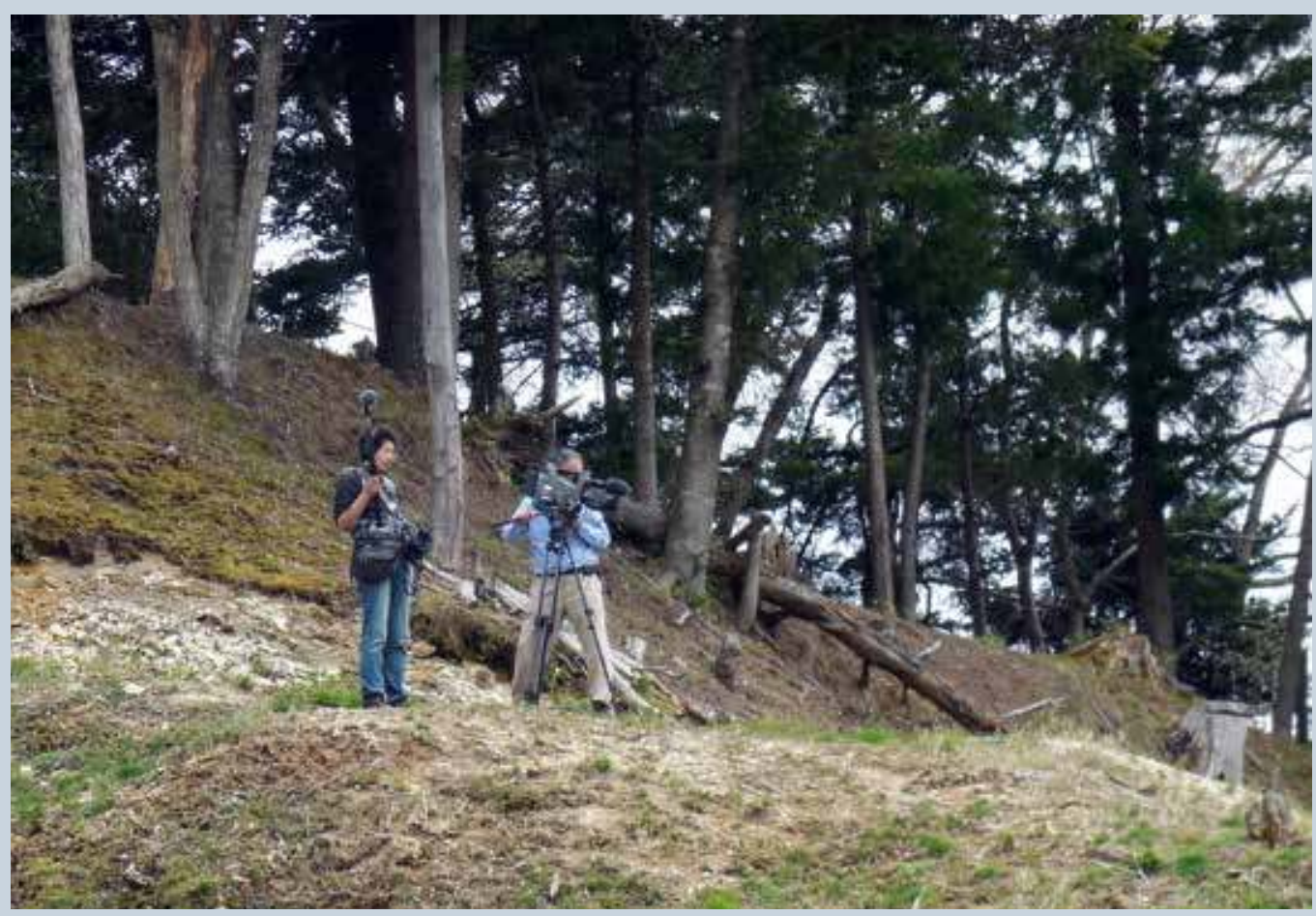


1999) nécessaire à l'appréhension de la vie en territoire contaminée. Instrument destiné à rendre «visible» et « audible» la présence des radioéléments, le compteur agit à la fois comme un révélateur d'événements chaotiques et comme un lanceur d'alerte. De la même manière qu'on a donné au gaz une odeur (on dit qu'il est «additivé»), l'histoire scientifique a pourvu la radioactivité d'un son caractéristique aisément reconnaissable, qui peut aider à aiguiser notre vigilance sur ce terrain délicat. Tant par le fil que je poursuis - comment se rendre sensible à la présence de la radioactivité - que par l'écriture qui en fait le récit, je chercherai ainsi à restituer la texture propre à ce territoire dorénavant peuplé d'entités sans présence.

\section{"Voir une fois au lieu d'écouter cent fois "}

Novembre 2012. Retour en arrière. Je venais alors pour la première fois dans la région de Fukushima, suivant l'appel - entre injonction et invitation - à «aller voir» que m'avait adressé Keiichi Ishii, professeur de sociologie économique à la faculté d'Agriculture de Sendai, Japon. Spécialiste des politiques agricoles, Keiichi avait d'abord travaillé en Europe avant de s'intéresser au développement de l'agriculture biologique japonaise. Il se trouve que le département de Fukushima, devenu tristement célèbre après le triple événement du 11 mars 2011, constituait jusqu'alors le creuset d'une agriculture biologique florissante. Keiichi connaissait bien les agriculteurs et les réseaux d'agriculteurs et se retrouvait ainsi aux premières loges pour suivre les reconfigurations des pratiques agricoles induites par la présence irréversible des radionucléides dans l'environnement. "Voir une fois au lieu d'écouter cent fois, selon une maxime japonaise ${ }^{3}$ ", m'avait-il écrit dans le message qu'il m’adressait avant ma venue. Il m’avait ainsi laissé supposer que pour comprendre, il fallait d'abord prendre par soi-même la mesure de la situation, il fallait se rendre témoin oculaire de ce qui était advenu de ce territoire dorénavant isolé.

Nous nous apprêtions à aller rencontrer Monsieur Nemoto, que Keiichi me décrivit comme un des leaders du village. Monsieur Nemoto avait longtemps dirigé la coopérative agricole et même s'il n'occupait plus cette fonction, c'était plus ou moins toujours lui, le chef. Il avait 75 ans. Aux dires de Keiichi, Ôdaka, où habitait Monsieur Nemoto, et ses alentours constituaient, dès avant la catastrophe, une zone économiquement fragile. Et avant même la catastrophe, la zone connaissait un vieillissement inquiétant de la population. Beaucoup de gens de la région travaillaient pour la centrale, qui se montrait généreuse en subventions en tous genres. La mise en doute de ce qui s'y passait constituait donc un point délicat. Si les agriculteurs s'étaient globalement élevés, dans les années 1970, contre l'installation puis le développement des centrales de Fukushima Daiichi et Fukushima Daini, force était de reconnaître que leurs arguments n'avaient pas emporté gain de cause. Et bien entendu, en 2012, il était plus aisé de formuler, rétrospectivement, les arguments d'une réticence qui n'avait pourtant plus lieu d'être... Située au plus proche de la zone interdite, dix kilomètres entourant la centrale de Fukushima Daiichi, Ôdaka avait été relativement épargnée des retombées radioactives - le vent avait été leur allié. Si les doses n'étaient, selon Keiichi, 
«pas très élevées», la proximité physique d'avec la centrale continuait cependant d'engendrer un sentiment d'insécurité. Les villages comme Ôdaka s'étaient retrouvés divisés: certains habitants ne projetaient plus de revenir vivre chez eux, tandis que d'autres souhaitaient reprendre leur activité agricole au plus tôt. Le projet auquel Keiichi participait consistait à accompagner, pour ceux qui en avaient le désir, ce retour à la terre dorénavant porteuse de sa part d'étrangeté ${ }^{4}$. «Il faut qu'ils tiennent encore dix ans. Alors la dose aura réduit petit à petit, et les jeunes générations reviendront peut-être. » Le travail engageait donc une prospective sur le long terme, égrenée d'incertitudes et d'ombres portées notamment par le caractère capricieux - «stochastique » est le qualificatif scientifique - de ce qu'on appelle les «faibles doses».

Tous les gens de la région s'étaient vus distribuer des dosimètres, m’avait

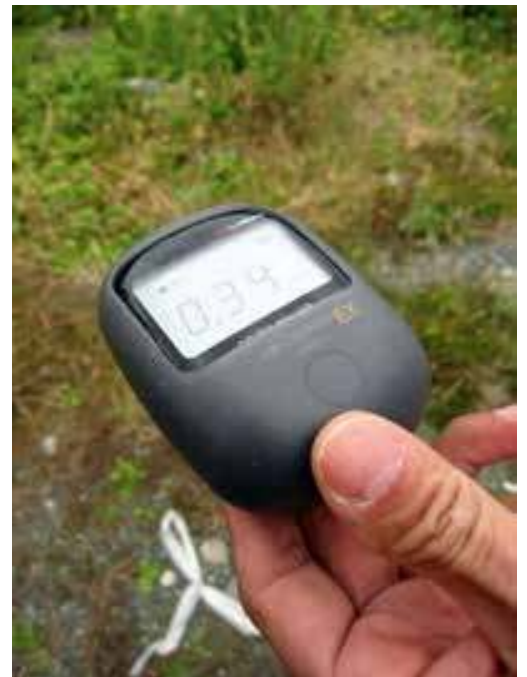

3. L'«Air Counter Ex» de Keiich dit Keiichi. Il en avait un également, qu'il avait obtenu par l'intermédiaire de son université. L'«Air Counter Ex» était un petit appareil que Keiichi posait entre nous dans la voiture et gardait sur lui quand nous en descendions. Tout au long de notre visite, je gardai un œil sur lui, captivée par ces chiffres qui croissaient et décroissaient parfois en quelques secondes. De l'autoroute, très rapidement au sortir de Sendai, nous commençâmes à apercevoir sur notre gauche les traces du tsunami. À l'horizon, à quelques kilomètres, une rangée d'arbres marquait le bord de mer. Entre la route où nous étions et le bord de mer maintenant visible, rien. Des étendues de terre retournée, où plus aucune habitation ni bâtiment ne se dressaient. Le dosimètre affichait 0.05. Puis 0.07. «Tant qu'on ne dépasse pas les 0.23 ou 0.24, me dit Keiichi, on est au-dessous des normes de sécurité. »Comme la plupart des gens que j’allais rencontrer, Keiichi avait parfaitement intégré la méthode de conversion qui permettait de décalquer les mesures, qui s'affichaient sur son compteur en micro-sieverts par heure, en dose normée sur l'année. Il effectuait ce calcul promptement sans discuter plus avant de la pertinence des seuils édictés comme acceptables et qui avaient changé avec le temps. Un peu plus au sud, sur la route, à la station routière où nous nous arrêtâmes pour déjeuner, le compteur afficha 0.13. Puis immédiatement 0.16. Puis 0.9. «Il faut toujours attendre un peu que le dosimètre se stabilise », commenta Keiichi. Parvenu dans la commune d'Ôdaka, Keiichi poursuivit jusqu'au bout de la route, arrêtée soudainement par le barrage des agents de police marquant l'entrée de la zone interdite. Avant de rebrousser chemin pour gagner la propriété de Monsieur Nemoto, le compteur affichait 0.19. Cette route, qui constituait un axe majeur reliant Sendai à Tôkyô, était dorénavant coupée par la zone interdite, tout comme la ligne de chemin de fer nationale que nous venions de longer (Jôbansen) ${ }^{5}$, transformant Ôdaka en «cul-de-sac». Nous nous arrêtâmes devant la maison de Monsieur Nemoto et Keiichi descendit voir s'il était prêt à nous recevoir. En l'attendant, je gardai un œil sur le compteur: $0.13,0.17,0.25,0.26,0.27,0.28,0.29,0.31,0.26 \ldots 0.41$. Nous étions arrivés un peu tôt pour notre rendez-vous avec Monsieur Nemoto, occupé avec quelqu'un d'autre. Keiichi me conduisit jusqu'aux parcelles d'expérimentation. À un croisement, une de 


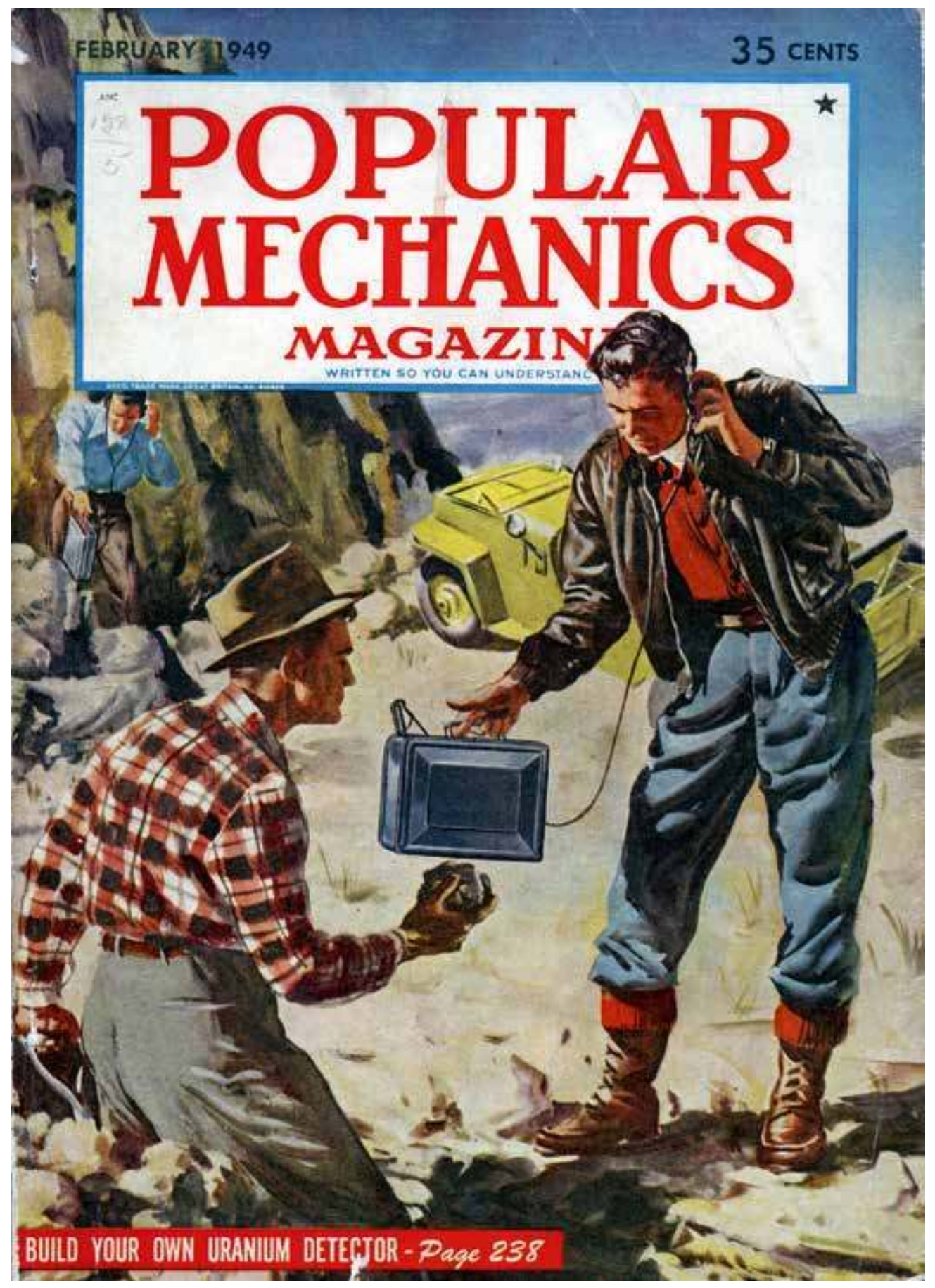

4. Prospecteurs d'uranium

tout à la joie d'entendre 
ces stations de mesure installées par le gouvernement au lendemain de la catastrophe indiquait 0.35. Quelques dizaines de mètres plus loin, sur une petite route au milieu des champs, un petit panneau indiquait l'emplacement de la parcelle expérimentale appartenant à Monsieur Nemoto. C'est là que trois années plus tard, j'entendrais le pic-vert.

Qu'avais-je vu alors en 2012, tandis que nous roulions de Sendai à Minami-sôma le long de la côte dévastée? J'avais vu des abords de route envahis par de hautes herbes, la mer à l'horizon maintenant dégagé, des amas de ferrailles tordues ici et là, des commerces fermés, des baraquements éventrés, des villages abandonnés. J'avais vu les chiffres du compteur Geiger de Keiichi monter et descendre à mesure que nous roulions, monter et descendre encore à mesure que nous arpentions l'habitation de Monsieur Nemoto, son jardin potager, ses parcelles. J'étais venue voir, mais au terme de ce premier séjour, j'étais repartie avec le sentiment que la vision, le regard, l'observation, ne suffiraient peut-être pas ou ne constitueraient pas les meilleures prises pour comprendre ni rendre l'importance de cette expérience - la mienne, pas plus que celle des autres. Et de fait, si, lors de ce premier séjour et lors des suivants, j'avais bien noté la présence et la médiation silencieuses du compteur Geiger, je ne pouvais que constater en 2015 qu'il avait disparu des interactions et déplacements quotidiens - celui de Keiichi remisé dans la boîte à gants de sa voiture.

\section{"First Lively Dance Music "}

Au regard des marques laissées par le tremblement de terre et le tsunami, l'empreinte laissée par l'accident nucléaire m'était donc apparue discrète au double sens du terme. Discrète, d'abord, en tant qu'elle s'opposait au spectaculaire, entrant de fait dans la catégorie des «aléas non synoptiques, diffus dans le temps et dans l'espace» (Moreau 2017: 96) qui imposent, au-delà de l'événementialité, d'étendre considérablement le spectre de l'analyse. Discrète ensuite, au sens mathématique du mot, parce que les chiffres révélaient une présence des radionucléides qui n'avait rien de continu, une manière chaotique de peupler l'espace - que les zones tracées sur les cartes étaient évidemment impropres à rendre. Sur le compteur de Keiichi, les chiffres qui s'affichaient, qui montaient et qui descendaient, me laissaient une impression confuse que ne résorberait pas la connaissance acquise des seuils, des conversions, des effets induits de la radioactivité. La «haute résolution» des stations de monitoring installées ici et là par le gouvernement pas plus que la «basse résolution» des compteurs Geiger individuels ne me permettaient de qualifier ce qui se passait là ni d'en prendre la pleine mesure. Pour les avoir entendus dans des films ou des documentaires, pour avoir lu les récits de commentateurs, j'avais en tête la sorte de grésillement caractéristique, un «crac crac» (Afchain et al. 1999) ou un «ça crache». Mais en désactivant la fonction sonore de l'appareil, mes interlocuteurs m'avaient paradoxalement rendue aveugle à la présence des radionucléides. Comment pouvais-je trouver à nouveau dans la machine de quoi donner de la consistance à mon expérience (Rancière 2014)? 
Lorsqu'Henri Becquerel publia en 1903 ses Recherches sur une propriété nouvelle de la matière, dans lesquelles il fit état des conclusions des expériences qu'il menait depuis 1896, il illustra son propos d'une série de soixante photographies qui attestaient visuellement pour la première fois des «mystérieuses émissions» de la radioactivité (cité dans Wilder 2011). Elles constituaient selon lui une «mise en évidence» qui rendait la radioactivité enfin «perceptible » et lui conférait une «matérialité» (ibid. : 352) ${ }^{6}$. Durant la décennie suivante, les efforts de la communauté scientifique portant sur la désintégration des particules radioactives (à l'origine de leur fameuse «émission ») portèrent sur des techniques destinées à compter combien d'atomes se transmutent par seconde. Jusque dans les années 1920, la technique qui dominait les expériences consistait ainsi à des scintillations produites par les particules désintégrées sur un écran fluorescent

Tenue entièrement voir, la méthode requérait cepentions d'observation contraignantes, pour rien dans les observateurs «rester dans le noir taine de minutes » leurs yeux, qui se de deux ou trois compté des scintilnombres (Hughes physicien Ernest

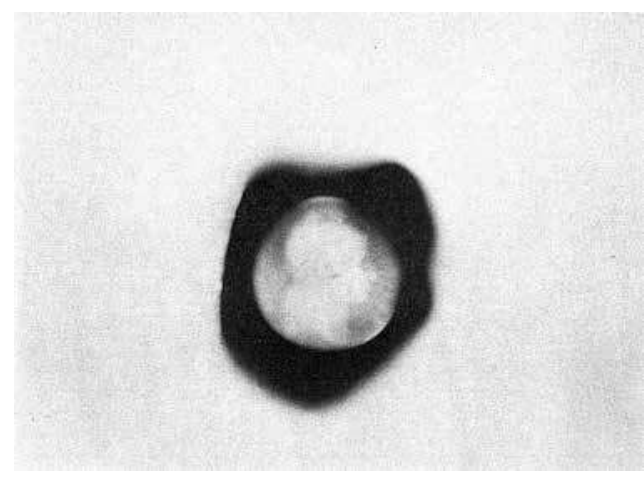
(Hughes 1998: 62). sous le régime du par scintillations dant des condiparticulièrement qui ne furent pas son abandon : devaient en effet pendant une vingafin d'accoutumer fatiguaient au bout minutes d'avoir lations en grands 1998: 64 citant le Rutherford). Parce qu'elles peinaient à être reproduites et stabilisées, parce qu'elles rendaient l'expérience dépendante des compétences visuelles d'observateurs toujours singuliers, ces observations furent progressivement remplacées, au cours des années 1920, par des méthodes de comptage électriques. Développées par Ernest Rutherford et Hans Geiger dès les années 1910 à Manchester, ces méthodes électriques consistaient à enregistrer l'ionisation produite par le passage d'une particule chargée au travers d'un gaz contenu dans un tube qui servait de « détecteur». Si le protocole n'était initialement guère plus stable que celui par scintillation, il bénéficia, dans les années 1920, du boom des techniques de radiodiffusion qui révélèrent son potentiel. Le développement et la commercialisation sans précédent des valves et autres composants électriques, fleurons de l'industrie radiophonique, gagnèrent les laboratoires de physique nucléaire qui virent là le moyen de déporter la sensibilité de l'observateur sur le dispositif lui-même. Profitant des techniques afférentes au «nouveau médium de consommation culturelle domestique» (Maisonneuve 2006) que représentait alors la radio, les physiciens des particules trouvèrent là un moyen, autrement plus efficace que les scintillations, d'« amplifier les petits courants électriques produits dans la chambre d'ionisation » (Abele 1999: 72). Autour de 1928, Geiger et Karl
5. Un médaillon radiographié à l'uranium qui illustre les Recherches sur une propriété nouvelle de la matière d'Henri Becquerel (1903) 
Müller équipèrent le compteur d'un haut-parleur qui impressionna profondément les auditeurs des congrès auxquels les deux hommes étaient invités - les « clics» "procur[ant] soudainement la sensation d'une perception immédiate des radiations» (ibid. : 82).

L'histoire dont je me plais ici à retracer les moments forts ne me permet pas de certifier que le dispositif auditif ne fut conçu qu'à la seule fin d'impressionner l'auditoire. Mais force est de reconnaître que l'équipement sonore de l'appareil ouvre à lui tout seul un champ d'appréhension inédit: il ne mesure pas plus qu'il ne compte, mais offre un indice de coexistence à haute valeur démonstrative et spectaculaire. Par un drôle revirement de la contagion technique, des compteurs furent même installés, au début des années 1960, sur des postes radios à usage domestique dans l'espoir que cette combinaison inédite mais somme toute naturelle - les uns et les autres reposant sur des dispositifs de captation de signaux retransmis acoustiquement facilite la popularité des instruments de mesure. L'innovation fut éphémère mais la presse fut prompte à célébrer le dispositif, susceptible, en cas de contamination quelle qu'elle soit, d'offrir à ses auditeurs une musique d'un genre nouveau, «the first lively dance music » jamais entendue (Abele 1999 : 98).

\section{Répertoires sensibles}

À l'automne 2016, je me rendais une nouvelle fois dans la région. Cette fois-ci, je venais accompagnée et équipée. Le besoin s'en était fait grandement sentir à mesure de mes séjours, qui m'avaient laissé l'étrange sensation de ne rien comprendre et la certitude que d'autres outils, d'autres regards, d'autres paradigmes me seraient nécessaires pour cerner ce qui véritablement importait dans ce dont je me rendais témoin. J'avais atteint un point de rupture dans l'enquête le jour où, au retour du Japon, je compris que mes notes de terrain, pourtant volumineuses, avaient passé sous silence un épisode qui m’avait bouleversé : autrement dit, je n'avais su consigner qu'une mineure partie de ce que j'avais d'abord vécu comme une expérience ${ }^{7}$. Je mettais alors à profit les collaborations établies durant les années précédentes avec des praticiens d'autres disciplines et auxquelles le collectif Call It Anything avait donné ses contours ${ }^{8}$. Ensemble, nous décidions de marcher. Nous décidions d'aller nous balader à Fukushima. Parmi les outils dont nous partions équipés: deux compteurs Geiger récemment acquis auprès de la CRIIRAD ${ }^{9}$, modèle «RADEX 1503 » conçu par un fabricant russe. Afin de se familiariser avec leur maniement, nous avions pris conseil auprès de Roland Desbordes, physicien de formation, qui dirige la CRIIRAD. Guidé par Roland, chacun de nous avait appris la séquence des gestes à effectuer: repérer le tube (au dos de l'appareil : le côté brillant) pour ajuster le compteur à l'objet à mesurer; allumer; paramétrer (choisir le seuil de détection, dans le menu alarme choisir avec ou sans son...); prendre d'abord le pouls de son environnement en mesurant le «bruit de fond» (qui correspond à la radioactivité naturelle, autour de 0.10 à Paris); attendre; poser sur l'objet à tester, le tube le plus près possible de l'échantillon; relancer le comptage; attendre. Attendre: «Quand 
le carré sur le côté devient clair, ça veut dire que la mesure est stable.» Roland nous avait mis en garde: le RADEX 1503 avait été configuré pour faire une mesure toutes les trente secondes. Sur cette base, il établissait des moyennes et parvenait ce faisant à stabiliser des valeurs. "Très léger », «très stable», mais «pas très rapide», avait commenté Roland, notre compteur ne nous donnerait rien de plus, si l'on allait trop vite, que de «petits signaux». Il nous faudrait donc «ne pas marcher trop vite». Huit jours durant, donc, nous marchâmeslentement: le long des routes de campagne aux alentours de la ferme où nous logions, à une cinquantaine de kilomètres de la centrale, dans une zone où les gens n'avaient pas été déplacés; nous marchâmes le long de la côte; nous marchâmes dans les forêts avoisinantes; nous traversâmes un village où les travaux de décontamination étaient en cours. Lors de ces déplacements, nous ne visions pas l'acquisition de données plus objectives ni une représentation plus légitime de la situation. Nous concevions plutôt la marche comme un geste instaurateur consistant à reproduire de la continuité dans le territoire distendu de Fukushima, et le compteur Geiger comme le révélateur potentiel de ses points de butée.

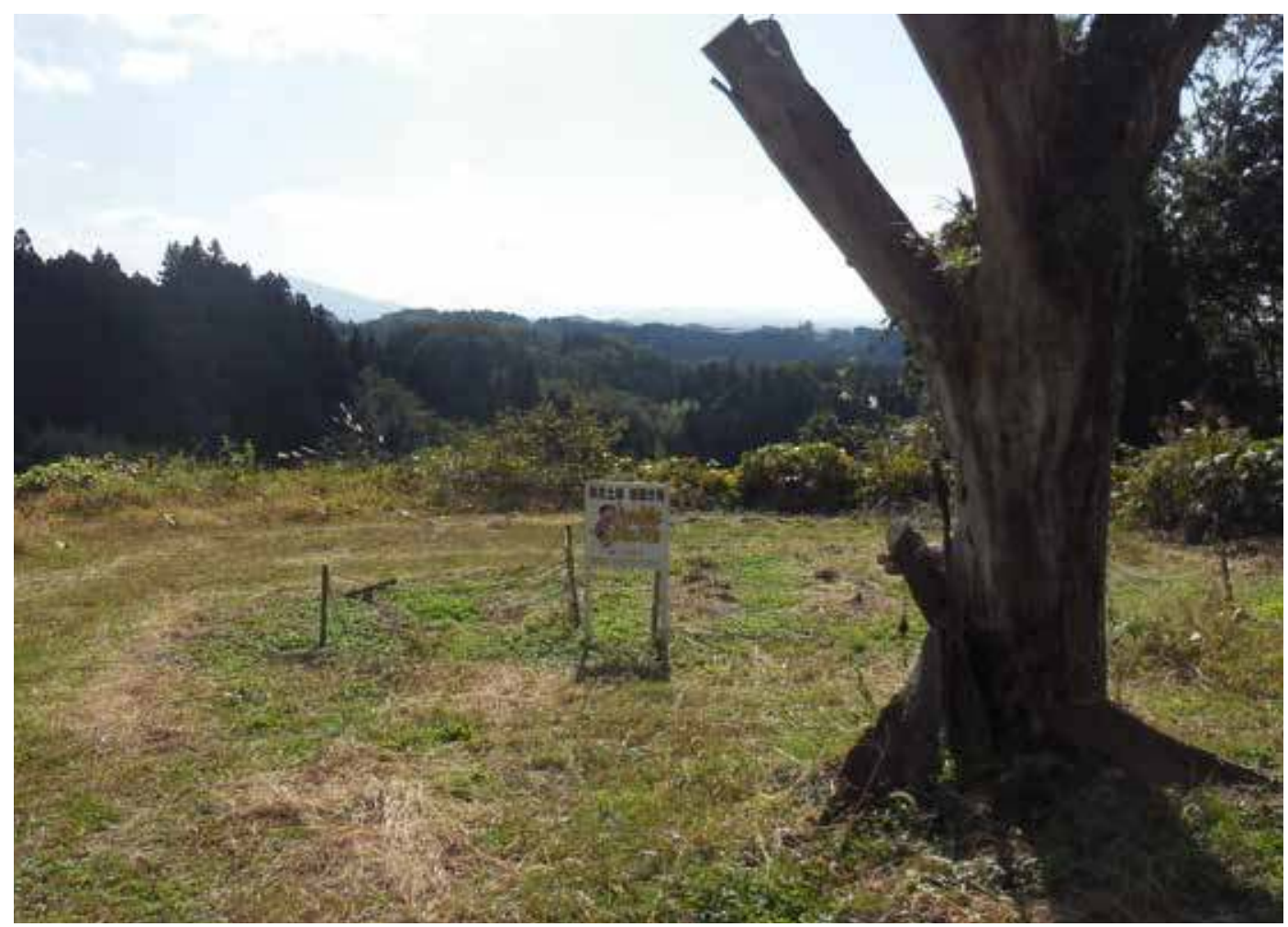

6. Un arbre au pied duquel avaient été déposés provisoirement des sacs de terre radioactive. Le panneau indique encore: «Dépôt de terre enlevée à cet endroit. Ne pas entrer!! » (Jokyo dojyô kari oki ba. Kono naka ni hairanaide!!). 
7. Balade à Yamakiya, en train d'être décontaminé. De dos, Yoann Moreau et Marc Boissonnade.

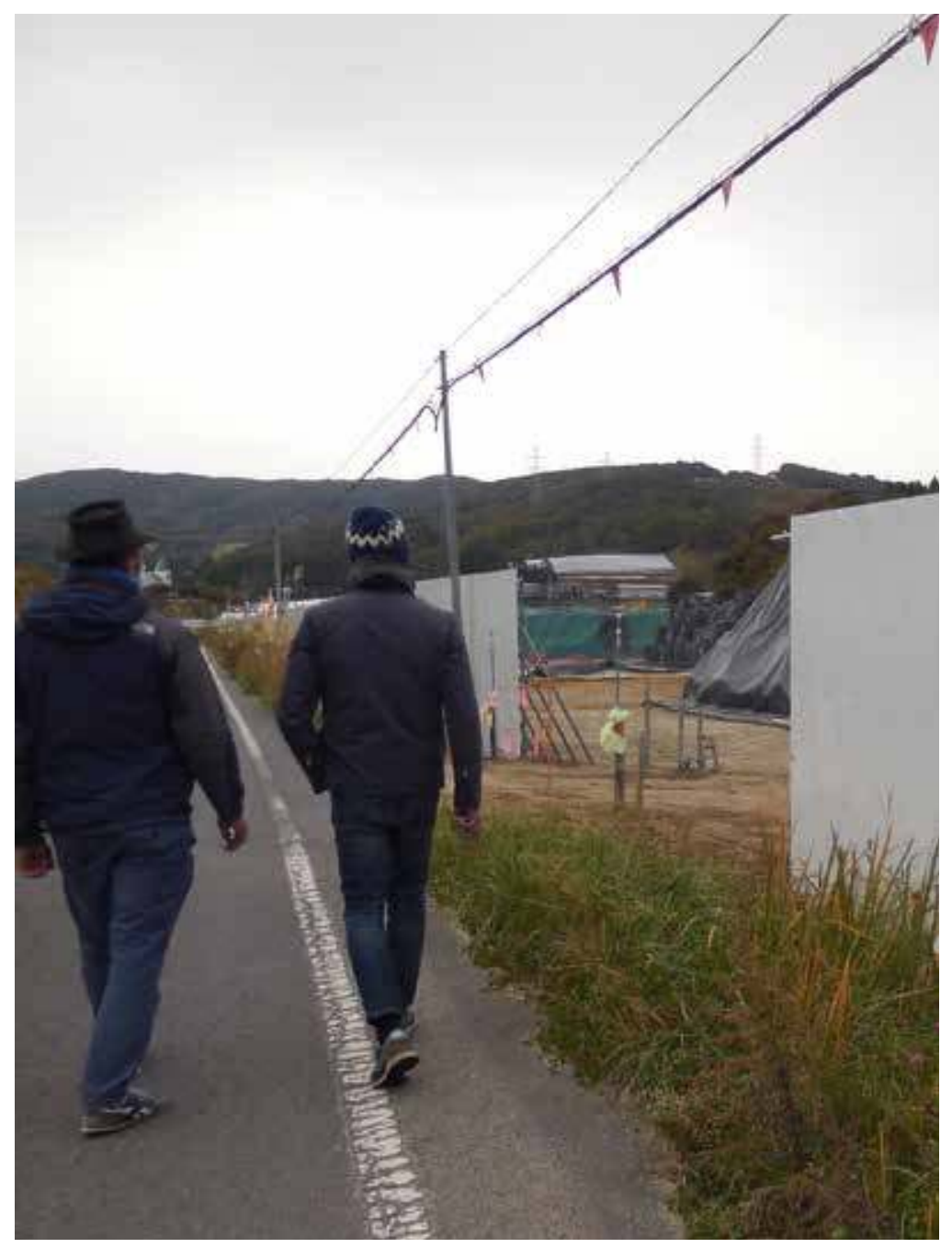

Au cours de nos balades, nous expérimentions à tour de rôle la marche avec le compteur. Le regard vissé à l'écran, certains s'arrêtaient pour tester, à certains endroits jugés propices au dépôt de radionucléides, leur présence: une rigole sur le bas-côté, le pied d'un arbre, un monticule herbeux, les abords d'un site où des sacs de déchets avaient été entassés. D'autres marchaient avec le compteur dans la poche, oubliant son existence jusqu'à ce qu'il émette de petits «bips» avec plus ou moins d'insistance. On avait appris grâce à Roland qu'à chacun de ces bips était corrélé le clignotement d'un petit carré noir dans la fenêtre du compteur, l'un et l'autre indiquant à sa façon la détection d'un «coup» ou «rayonnement». Ils ne mesuraient pas, ne contrôlaient pas mais signalaient et nous indiquaient subtilement que l'espace autour de nous était traversé de manière plus ou moins dense et continue d'événements aléatoires - de désintégrations d'atomes. Le bip constituait donc un effet sonore qui nous «donnait une idée» du type d'environnement dans lequel on circulait sans voir. Nous obtenions là bel et bien ce que j'ai appelé un indice de coexistence que paraissait soutenir une logique différente de celle de l'affichage chiffré: un chiffre bas au compteur n'était en effet pas contradictoire avec l'émission de «bips » sporadiques. Mais chaque «bip » nous donnait l'assurance que nous marchions en contiguité avec une «matière vibrante» (Bennett 2010) et émettrice.

La rencontre des rayons que signait chacune des émissions sonores ne nous préparait cependant pas totalement à l'expression de leur débordement. Ainsi ce jour où nous roulions sur la Route 6 et traversions la zone «de retour difficile»-doux euphémisme pour désigner ce qui ailleurs s'appelle «zone interdite». Parvenus à trois kilomètres de la centrale, dans l'habitacle du véhicule toutes fenêtres et bouches d'aération fermées, le compteur se mit à tenir, dans la gamme des aigus, des bips en continu. Dans la tonalité et le maintien de la note, 
nous avons entendu l'alarme et compris sans équivoque qu'il ne faisait pas bon être là. Nous nous sentions littéralement assaillis sans pouvoir nous défendre. Des rayons nous traversaient, étaient partout. Nous ne pouvions rien faire d'autre que rouler pour sortir de la zone au plus vite, et nous protéger en collant sur nos bouches masques ou écharpes. Leur présence était vive, soudain concentrée. Les radioéléments ne nous paraissaient plus amassés ici et là, au détour d'un chemin ou au pied d'un arbre, contenus ou déposés; ils ne s'exprimaient plus de manière aléatoire au gré de nos déplacements ou même de nos mouvements: ils n'étaient plus épars dans l'environnement - visqueux et insinuants, ils constituaient précisément ce dans quoi nous étions, nous privant de tout «ailleurs » (Morton 2013). Probablement augmentés encore par l'exiguité de l'habitacle, les bips prolongés et stridents officiaient ainsi soudainement ce pour quoi ils avaient été conçus: nous «connecter émotionnellement à quelque chose d'incompréhensible autrement » (Supper 2014: 47), transformer en sensation directe un phénomène impossible à acquérir d'expérience et permettre un «engagement sensoriel» (ibid.), viscéral même, qui ne pouvait que rendre poignante - au sens où l'on pourrait enfin s'en saisir - l'énormité de la situation.

Dans son ouvrage Hyperobjects, Timothy Morton écrit que «ce qui explique le mieux la conscience écologique est un sens de l'intimité: non le sentiment d'appartenir à quelque chose de plus grand mais celui d'être proche, trop proche même, d'autres formes de vie, de les avoir sous la peau» (Morton 2013: 139). Cette conscience requière selon lui de «s'accorder à», se mettre au diapason de ces entités qui nous peuplent en même temps qu'elles constituent notre milieu. Soudainement dépourvu de liants par le comportement stochastique des radionucléides, le territoire de Fukushima impose ainsi à celui qui s'en préoccupe de changer de répertoire sensible et de se mettre au diapason de ce qui dorénavant l'habite. C'est une partie de cet itinéraire que j'ai tenté de retracer ici en interrogeant et en cherchant à qualifier: «que se passe-t-il?». J'ai tenté de restituer la texture de ce monde-ci, en rendant compte -parce que c'est inextricable - de ces moments où j'ai compris que je ne pouvais plus connaître de la même manière, ces moments où le modèle même de l'enquête s'est avéré débordé de partout. Sur ce terrain contaminé, le régime d'expérience sensible est comme suspendu et cette suspension laisse la place à d'autres régimes d'appréhension du réel qui requièrent un appareillage (le compteur Geiger) et des dispositifs (la balade collective, l'écriture en récits) spécifiques. Dans la région de Fukushima, l'environnement a bel et bien changé de tonalité. Et si tant est qu'on maximise ce dont il est capable, le compteur Geiger constitue l'outil idoine pour apprécier un tel changement: non parce qu'il fournit l'assurance objective de ce dont on se porte témoin en circulant sur le territoire de Fukushima, mais parce qu'il compte au nombre des pratiques d'écoute privée au moyen desquelles chacun peut à loisir amplifier les bruits du monde - et en l'occurrence, du monde physique lui-même. 


\section{Notes}

1. Les données mobilisées dans cet article sont issues de plusieurs missions de terrain qui se sont égrenées entre 2012 et 2016 dans le département de Fukushima. Ces missions ont été soutenues par le Laboratoire d'ethnologie et de sociologie comparative (CNRS, UMR7186) ainsi que par le centre de culture scientifique, technique et industrielle F93, à Montreuil. Je remercie en particulier Marc Boissonnade, directeur de F93, d'avoir permis un déploiement inédit de ces missions et d'assumer si pleinement leur charge exploratoire.

2. Dépendant du cabinet du Premier Ministre, l'«Agence pour la Reconstruction» (Fukkô-chô) voit le jour en février 2012. Le projet «Grand Design» (Gurando Dezaiin) s'attèle à la remise en œuvre de la vie, sous tous ses angles, dans le département de Fukushima.

3. L'expression japonaise que Keiichi traduit à mon intention est probablement (hyakubun wa ikken ni oyobazu) que l'on pourrait aussi traduire par «Cent paroles ne valent pas un regard».

4. Uncanny Terrain est le titre d'une série documentaire produite par Junko Kajino et Ed Koziarski qui explore les multiples facettes de ce retour à la terre: uncannyterrain.com/blog/. Pour une analyse de la série, voir Prignot 2015.

5. En plus de constituer jusqu'à la catastrophe un axe de circulation majeur entre les deux grandes villes de Sendai et de Tôkyô, la Route Nationale 6 longe plusieurs centrales nucléaires, donc Tôkaimura, la première à avoir été implantée au Japon. Un peu à la manière de la route des vins en France, elle est de ce fait connue comme «la route des centrales». Traversant la zone interdite, elle a réouvert à la circulation à l'automne 2014 : il est à présent possible de l'emprunter mais il est interdit de s'arrêter, sortir de sa voiture, et toutes les routes adjacentes restent barrées. La ligne ferroviaire a quant à elle réouvert partiellement en 2016.

6. Ces images appartiennent à une longue histoire de l'instrumentation destinée à voir ou donner corps aux infimes composants de notre environnement. Voir notamment Crary 1992, Daston \& Galison 2012, Keller 2008, Galison 1997, Galison \& Jones 1998.

7. Je tente de donner ses premiers contours à cette expérience dans Houdart 2015.

8. Formé courant 2012 à l'initiative de Marc Boissonnade, F93, ce collectif a connu plusieurs configurations à ce jour (www.f93.fr/fr/project/11/ call-it-anything.html). L'épisode relaté ici a constitué à la fois une nouvelle phase d'enquête collaborative et un nouvel assemblage composé de Marc Boissonnade, Stéphane Sautour (artiste plasticien), Patricia Falguières (historienne de l'art), Mélanie Pavy (réalisatrice de films), et Yoann Moreau (anthropologue et metteur en scène). Myriam Lefkowitz (danseuse et chorégraphe) et Vinciane Despret (philosophe) ont accompagné le projet à distance.

9. La Commission de Recherche et d'Information Indépendantes sur la Radioactivité est une association fondée en France en 1986, au lendemain de la catastrophe de Tchernobyl.

\section{I'auteure}

Sophie Houdart est anthropologue, directrice de recherche au CNRS, Laboratoire d'ethnologie et de sociologie comparative (LESC). Spécialisée sur le Japon, elle a réalisé plusieurs enquêtes sur le thème de la création et de l'innovation, dans les champs des sciences, de l'art ou de l'architecture. Elle travaille aujourd'hui sur la vie après la catastrophe de Fukushima.

\section{Iconographie}

Image d'ouverture. Dans la cour de Monsieur Nemoto, un faisan picore. () Sophie Houdart.

Crédits iconographiques: (C) Sophie Houdart pour

4. Source: Popular Mechanics Magazine, 1949.

5. (C) Wellcome Library. toutes les images sauf: 


\section{Références}

Abele, J. 1999 «Safety cliks: The Geiger-Müller tube and radiation protection in Germany, 1928-1960» in R. Bud, B. Finn \& H. Trischler (dir.) Manifesting Medecine. Bodies and Machines. Netherlands Harwood academic publishers: 79-104.

Afchain, Y., Pauck-Bosc, C. \& H. Schertz 1999 La radioactivité. Paris: PEMF : 26-29.

Bennett, J. 2010 Vibrant Matter: A Political Ecology of Things. Durham \& Londres: Duke University Press.

Crary, J. 1992 Techniques of the Observer: On Vision \& Modernity in the Nineteenth Century. Cambridge: MIT Press.

Daston, L. \& P. Galison 2012 [2007] Objectivité. Paris: Les Presses du réel.

Debaise, D. 2015 L'appât des possibles. Reprise de Whitehead. Paris: Les Presses du réel.

Dettelbach, M. 1999 «The face of nature: Precise measurement, mapping, and sensibility in the work of Alexander von Humboldt », Studies in History and Philosophy of Biological and Biomedical Sciences 3(4): 473-504.

Filine, A. (collectif) 2012 Oublier Fukushima. Textes et documents. Le Mas d'Azil: Les éditions du bout de la ville.

Furukawa, H. 2013 [2011] Ô chevaux, la lumière est pourtant innocente. Paris: Picquier \& Protière.

Galison, P. 1997 Image and Logic: A Material Culture of Microphysics. Chicago: University of Chicago Press.

Galison, P. \& C. Jones 1998 Picturing Science, Producing Art. Cambridge: Routledge.

Haraway, D. 2010 [2002] Manifeste des espèces de compagnie. Chiens, humains et autres partenaires. Paris: Terra Incognita / Éditions de l'Éclat.

- 2016 Staying with the Trouble: Making Kin in the Chthulucene. Durham, Londres: Duke University Press.
Houdart, S. 2015 « Route $6 »$ in E. Grimaud \& A.-C. TaylorDescola, Persona. Étrangement humain. Paris: Actes Sud / Musée du quai Branly: 71-72.

Hughes, J. 1998 « Plasticine and valves: Industry, instrumentation and the emergence of nuclear physics» in J.-P. Gaudillière \& I. Löwy (dir.) The Invisible Industrialist: Manufacture and the Construction of Scientific Knowledge. UK: Palgrave Macmillan: 58-101.

Keller, C. (dir.) 2008 Brought to Light: Photography and the Invisible, 1840-1900. San Francisco: The San Francisco Museum of Art.

Maisonneuve, S. 2006 « De la machine parlante au disque. Une innovation technique, commerciale et culturelle», Vingtième Siècle. Revue d'histoire 4(92): 17-31.

Moreau, Y. 2017 Vivre avec les catastrophes. Paris: Puf.

Morton, T. 2013 Hyperobjects: Philosophy and Ecology after the End of the World. Minneapolis, Londres: University of Minnesota Press.

Prignot, N. 2015 «Malgré Fukushima. Récits de cultures improbables » in D. Debaise \& I. Stengers (dir.) Gestes spéculatifs. Paris: Les Presses du réel: 73-86.

Rancière, J. 2014 Le fil perdu. Essai sur la fiction moderne. Paris: La Fabrique.

Stengers, I. 2014 «Penser à partir du ravage écologique» in E. Hache (dir.) De l'univers clos au monde infini. Bruxelles: Éditions Dehors.

Supper, A. 2014 «Sublime frequencies: The construction of sublime listening experiences in the sonification of scientific data », Social Studies of Science 44(1): 34-58.

Tsing, A.L. 2015 The Mushroom at the End of the World: On the Possibility of Life in Capitalist Ruins. Princeton: Princeton University Press.

Wilder, K. 2011 «Visualizing radiation: The photographs of Henri Becquerel» in L. Daston \& E. Lunbeck (dir.) Histories of Scientific Observation. Chicago: The University of Chicago Press: 349-368.

\section{Pour citer l'article}

Houdart, S. 2017 «Les répertoires subtils d'un terrain contaminé», TechniquesE Culture 68 « Mondes infimes», p. 88-103. 